\title{
Inovação tecnológica no processamento de pães: otimização da incorporação de suco de acerola e manga
}

\author{
R. A. ZAMBELLI' ${ }^{1}$, M. M. N. ARAÚJO', S. C. P. MELO ${ }^{1}$, L. I. F. PINTO' ${ }^{1}$, P. E. F. MELO'1 e \\ D. F. PONTES ${ }^{1}$. \\ ${ }^{1}$ Universidade Federal do Ceará, Departamento de Tecnologia de Alimentos. \\ E-mail para contato: Zambelli@alu.ufc.br
}

\begin{abstract}
RESUMO - O estudo tem como objetivo utilizar o delineamento composto central rotacional na otimização na incorporação de sucos frutas em pães tipo forma. Foram definidas como variáveis independentes: os percentuais de suco de acerola e manga, as quais foram adicionadas em níveis variando de $0 \%$ a $50 \%$ e $0 \%$ a $50 \%$, respectivamente. Através da metodologia de superfície de resposta foram avaliados os efeitos sobre as características físicas e fermentativas das massas e pães: fator de expansão máximo, volume produzido e específico, densidade e índice de expansão. Para o fator de expansão, densidade e índice de expansão os valores ótimos estão em torno de $15 \%$ da adição de sucos, para volume produzido e específico os valores estão em torno de $19 \%$ da adição. Quanto maior à quantidade de suco empregada, maior foi a redução nas capacidades de expansão das massas. As condições ótimas encontradas foram: incorporação de até $16 \%$ de acerola e $18 \%$ de manga.
\end{abstract}

\subsection{INTRODUÇÃO}

A possibilidade de introduzir novos ingredientes para fabricação de pães com objetivo de melhorar as características do produto final é de interesse industrial, podendo significar produtos de melhor qualidade sensorial, nutricional e de maior valor agregado. Sucos de frutas são considerados produtos alimentícios saudáveis, são atualmente consumidos com frequiência por uma grande porcentagem da população mundial. $\mathrm{O}$ crescimento significativo no mercado de frutas tem atraído a atenção de produtores, distribuidores e processadores para atender as demandas (Renuka et al., 2009).

A acerola (Malpighia emarginata DC.) é um fruto tropical de grande valor nutritivo devido ao seu alto teor de vitamina C (1000-4000 mg/100 g de fruto) e pela presença de outros nutrientes, como as antocianinas, carotenóides, ferro e cálcio (Mercali et. al, 2014). Musser et al. (2004) avaliaram o teor de antocianinas de acerolas maduras a partir de 12 genótipos, os resultados mostraram uma variação média entre 3,8 e 47,4 mg/100 g. Estudos realizados para a identificação de pigmentos antocianinas em polpa de acerola descobriram que a fruta exibiu dois tipos de antocianinas: cianidina-3-rhamnoside e pelargonidina-3rhamnoside (De Rosso et al., 2008). A manga (Mangífera Indica L.) é uma das frutas tropicais mais apreciadas, tem sabor e aroma diferenciados, além de boa qualidade nutricional (Ahmed et al., 2005). Os frutos contém excelente quantidade de fibras, contendo cerca de 2,53,0 vezes mais conteúdo de beta-caroteno do que outras frutas (Singh e Singh, 2010).

O estudo tem como objetivo estudar a utilização do suco de acerola e manga em substituição a água em formulações de pães tipo forma utilizando o delineamento composto central rotacional e estudar o efeito nos parâmetros físicos de qualidade dos pães. 


\subsection{METODOLOGIA}

\subsection{Delineamento Experimental}

O desenvolvimento das formulações de pães tipo forma foi realizado a partir de uma formulação padrão, cuja composição é: $100 \%$ de farinha de trigo; $55-60 \%$ de água, $10 \%$ de Gordura Vegetal Hidrogenada; 5\% de açúcar, 3,3\% de fermento biológico e 2\% de sal.

$\mathrm{Na}$ tabela 1 são apresentados as faixas de valores codificados e reais utilizados para os ensaios e na tabela 2 é apresentada a matriz do planejamento.

Tabela 1 - Variáveis e níveis do planejamento experimental completo $2^{2}$

\begin{tabular}{cccccc}
\hline & \multicolumn{4}{c}{ Níveis codificados e reais das variáveis independentes } \\
\cline { 2 - 6 } Variáveis Independentes & $\mathbf{- \alpha}=\mathbf{- 1 , 4 1}$ & $\mathbf{- 1}$ & $\mathbf{0}$ & $\mathbf{+ 1}$ & $\mathbf{+ \alpha}=\mathbf{+ 1 , 4 1}$ \\
Suco de Acerola (\%) & 0 & 12,5 & 25 & 37,5 & 50 \\
Suco de Manga (\%) & 0 & 12,5 & 25 & 37,5 & 50 \\
\hline
\end{tabular}

*Em substituição à quantidade de água incorporada.

Para o desenvolvimento das formulações de pães tipo forma incorporadas com diferentes quantidades de suco de acerola e manga foi utilizado o Delineamento Composto Central Rotacional (DCCR). Foi aplicado um planejamento fatorial $2^{2}$ completo, totalizando 11 ensaios, sendo quatro fatoriais (combinação dos níveis -1 e +1 ), quatro axiais $(-\alpha$ e $+\alpha)$ e três repetições no ponto central (0) para estimativa do erro padrão.

Tabela 2 - Matriz do delineamento experimental com valores codificados e reais

\begin{tabular}{ccccc}
\hline Ensaios & $\begin{array}{c}\text { Suco de } \\
\text { Acerola }\end{array}$ & $\begin{array}{c}\text { Suco de } \\
\text { Manga }\end{array}$ & $\begin{array}{c}\text { Suco de Acerola } \\
(\boldsymbol{\%})\end{array}$ & Suco de Manga (\%) \\
\hline $\mathbf{1}$ & -1 & -1 & 12,5 & 12,5 \\
$\mathbf{2}$ & +1 & -1 & 37,5 & 12,5 \\
$\mathbf{3}$ & -1 & +1 & 12,5 & 37,5 \\
$\mathbf{4}$ & +1 & +1 & 37,5 & 37,5 \\
$\mathbf{5}$ & $-1,41$ & 0 & 0,00 & 25,0 \\
$\mathbf{6}$ & $+1,41$ & 0 & 50,0 & 25,0 \\
$\mathbf{7}$ & 0 & $-1,41$ & 25,0 & 0,00 \\
$\mathbf{8}$ & 0 & $+1,41$ & 25,0 & 50,0 \\
$\mathbf{9}$ & 0 & 0 & 25,0 & 25,0 \\
$\mathbf{1 0}$ & 0 & 0 & 25,0 & 25,0 \\
$\mathbf{1 1}$ & 0 & 0 & 25,0 & 25,0 \\
\hline
\end{tabular}

\subsection{Processamento dos Pães Tipo Forma}

Os ingredientes foram pesados em balança semi-analítica separadamente. Aplicou-se o método direto, onde todos os ingredientes são colocados simlutaneamente no início da etapa de mistura, com exceção do sal e água. Eles foram misturados em misturadora de escala semiindustrial durante 1 minuto em baixa velocidade para a homogeneização dos ingredientes, em seguida foi adicionada a água e misturada por 3 minutos em velocidade média, por último foi adicionado o sal e a massa foi misturada em alta velocidade por 6 minutos até o seu completo 
desenvolvimento. As massas foram divididas em porções de $250 \mathrm{~g}$ e moldadas na forma de elipses manualmente. Foram colocadas em fôrmas de folha galvanizada de ferro de chapa única para pão de forma sem tampa. Em seguida, colocadas em câmara de fermentação regulada a temperatura de $28{ }^{\circ} \mathrm{C} \pm 2{ }^{\circ} \mathrm{C}$ e $80 \%$ de umidade relativa, durante uma hora e trinta minutos. Ao final da fermentação, as massas foram assadas sem vapor durante 20 minutos a temperatura de $220{ }^{\circ} \mathrm{C}$ em forno elétrico de lastro Continental Advance Turbo®. Os pães foram resfriados durante uma hora em temperatura ambiente.

\subsection{Avaliação Física das Massas e dos Pães Tipo Forma}

Avaliação do processo de fermentação pela técnica de perfil livre de expansão: A expansão das massas durante o processo de fermentação foi avaliada através do procedimento experimental de Perfil Livre de Expansão desenvolvido por Gabric et al. (2011), com modificações, onde as massas foram consideradas como elipses truncadas, conforme ilustra a figura 1, foram medidas, a cada 10 minutos, as dimensões com o auxílio de régua milimetrada simples. As massas foram colocadas em fôrmas de alumínio com dimensões de $30 \mathrm{~cm}$ x 30 $\mathrm{cm}$ para permitir a sua livre expansão sem restrições dimensionais.

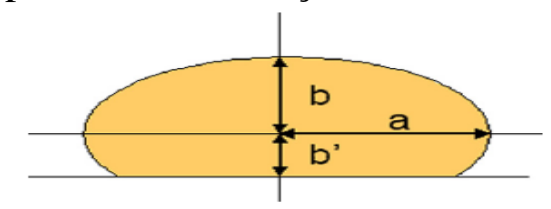

Figura 1 -Parâmetros de elipse truncada

O volume das massas para cada tempo de medição foi calculado com base na fórmula da elipse e sua rotação em torno do eixo y, conforme demonstra a equação 2.

$V\left(c m^{3}\right)=\pi a^{2}\left(\frac{2 b}{3}+b^{\prime}-\frac{b^{3}}{3 b^{2}}\right)$

Onde: $\mathrm{a}=$ largura da elipse $(\mathrm{cm}) ; \mathrm{b}=$ altura da elipse - do centro a elipse superior $(\mathrm{cm})$; $b^{\prime}=$ altura da elipse - do centro para a parte inferior $(\mathrm{cm})$.

O fator de expansão do volume $\left(\mathrm{cm}^{3}\right)$ - VEF, foi calculado através da equação 3 .

$V E F=\frac{V-V_{o}}{V_{o}}$

Onde: $\mathrm{V}=$ Volume da massa ao longo do tempo de fermentação $\left(\mathrm{cm}^{3}\right) ; \mathrm{V}_{\mathrm{o}}=$ Volume incial da massa $\left(\mathrm{cm}^{3}\right)$.

Volume Específico: As massas dos pães foram determinadas em balança semi-analítica e expressa em gramas. $\mathrm{O}$ volume deslocado dos pães foi medido através de preenchimento de recipiente plástico e transparente com semente de painço expressando o resultado $\mathrm{mL}$. $\mathrm{O}$ volume específico foi calculado pela divisão do volume deslocado do pão (mL) pela sua massa (g), segundo método ${ }^{\circ}$ 72-10 da AACC (1995).

Densidade: A densidade foi calculada através da relação inversa entre o volume deslocado e o peso da amostra assada. 
Índice de Expansão: Para o cálculo do índice de expansão foi adaptada a medotologia utilizada por Silva et al. (2003) para pães de queijo. As massas foram moldadas na forma esférica para permitir as medições do diâmetro e da altura. com auxílio de régua milimetrada simples. O Índice de Expansão (IE) dos pães foi calculado através da equação 4:

Índice de Expansão $(I E)=\frac{\frac{\left(D_{p}+H_{p}\right)}{2}}{\frac{\left(D_{m}+H_{m}\right)}{2}}$

Onde: $\mathrm{D}_{\mathrm{p}}$ e $\mathrm{H}_{\mathrm{p}}=$ Diâmetro e altura dos pães após o forneamento (cm); $\mathrm{D}_{\mathrm{m}}$ e $\mathrm{H}_{\mathrm{m}}=$ Diâmetro e altura das massas moldadas $(\mathrm{cm})$.

\subsection{Análise Estatística}

A avaliação dos resultados dos parâmetros físicos no desenvolvimento das formulações através do delineamento composto central rotacional foi realizada por superfície de resposta e suas respectivas curvas de contorno após a análise de variância (ANOVA) e regressão, foi realizado teste de médias ao nível de $5 \%$ de significância. A análise foi realizada no programa STATISTICA 7.0.

\subsection{RESULTADOS E DISCUSSÃO}

A figura 2 apresenta a superfície de resposta e curva de contorno para o fator de expansão das massas adicionadas de suco de manga e acerola.
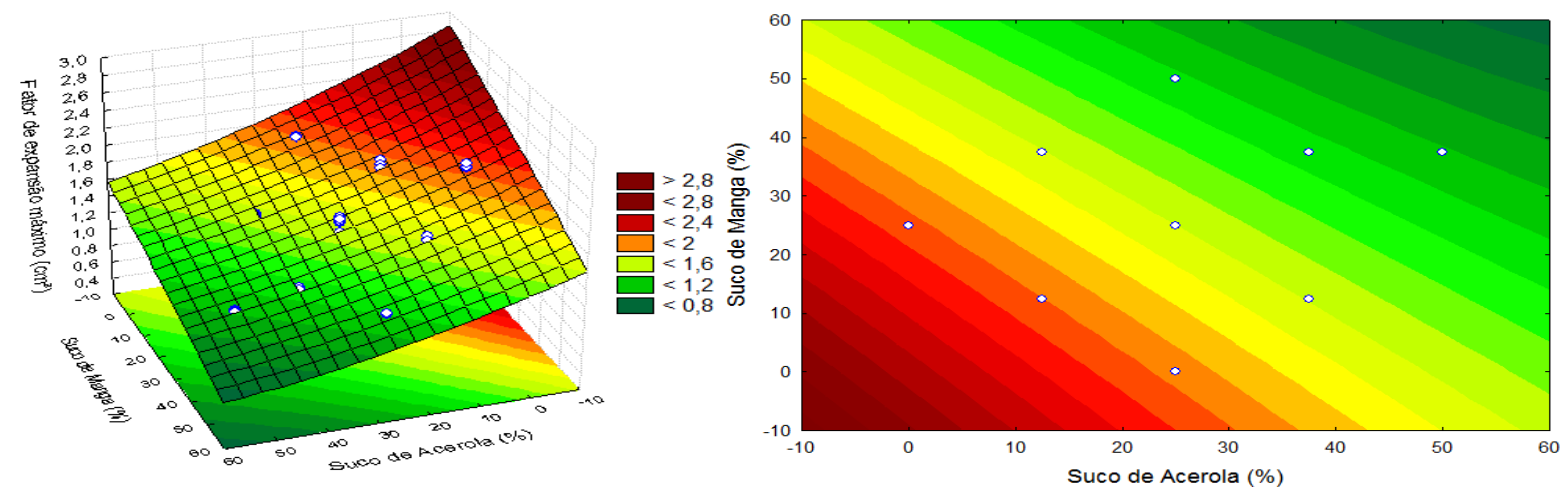

Figura 2 - Superfície de resposta, curva de contorno da ação do suco de acerola e manga sobre o fator de expansão máximo da massas

A zona de maximização do fator de expansão das massas compreendeu na substituição da água por suco de manga até $25 \%$ e de $15 \%$ para o suco de acerola, a região compreende valores superiores a 2,00 $\mathrm{cm}^{3}$. O ensaio 5 apresentou o maior valor, $2,06 \mathrm{~cm}^{3}$ e o 6 , com apenas $1,12 \mathrm{~cm}^{3}$, quando foi adicionado $50 \%$ de suco de acerola e $25 \%$ de suco de manga. Amaya et al. (2013a) estudaram a incorporação de suco de caju no fator de expansão das massas, quando a água foi substituída em $50 \%$, o valor obtido foi de $0,99 \mathrm{~cm}^{3}$ e $0,68 \mathrm{~cm}^{3}$ quando a substituição foi total, valores inferiores obtidos no estudo com o uso combinado de suco de acerola e manga. 
A redução do fator de expansão das massas pode ser explicado pela provável inviabilização de parte da levedura pela acidez dos sucos e também por danos causados à matriz do glúten pela presença de fibras, que segundo Wang et al. (2002) elas podem ligar-se à matriz protéica e causar pequenas fissuras, permitindo com que a matriz retenha menor quantidade de gás carbônico produzido durante o processo de fermentação das massas.

A figura 3 fornece a superfície de resposta e curva de contorno da ação dos sucos de acerola e manga no volume específico dos pães.
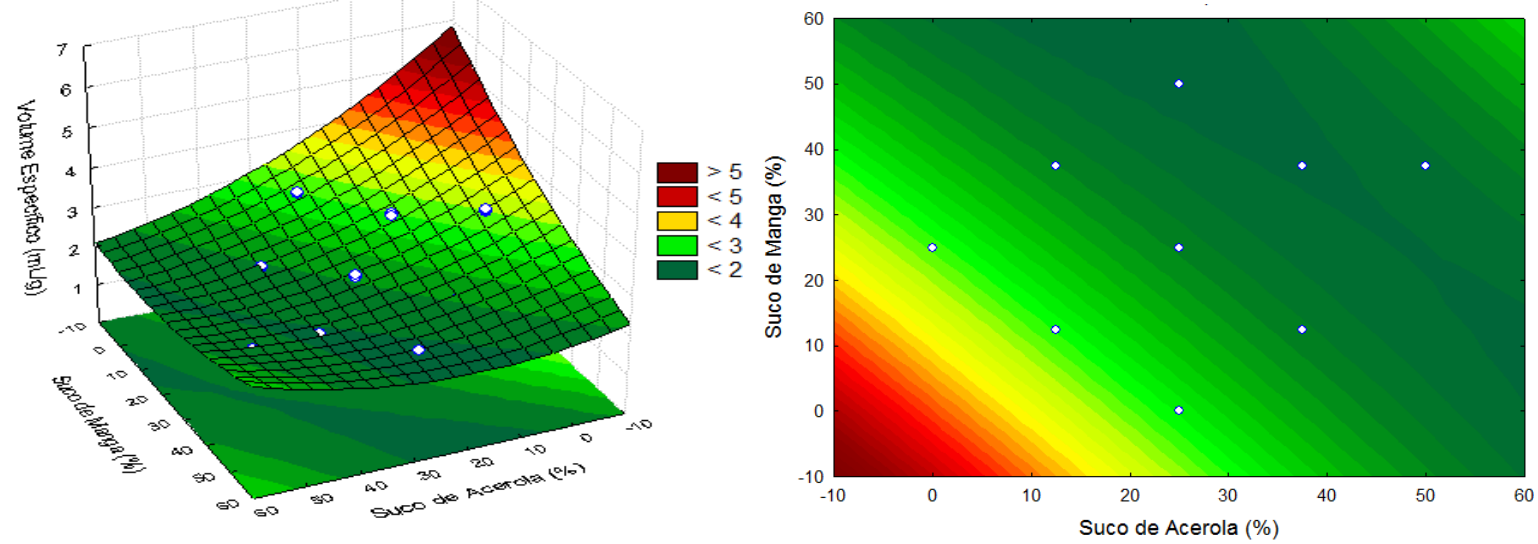

Figura 3 - Superfície de resposta, curva de contorno da ação do suco de acerola e manga sobre o volume específico dos pães

A adição de quantidades inferiores a $25 \%$ de suco de manga e $20 \%$ de suco de acerola promoveu volumes específicos superiores a $3,00 \mathrm{~mL} / \mathrm{g}$, o maior valor foi observado para o ensaio 7, com 3,04 mL/g, quando houve a adição de $25 \%$ de suco de acerola, sem a incorporação de suco de manga e o menor volume específico foi apresentado pelo ensaio 3 e 4 com 2,05 mL/g e 2,03 mL/g, que correspondem à quantidades de $12,5 \%$ a $37,5 \%$ de incorporação dos sucos. Valores inferiores a $2,00 \mathrm{~mL} / \mathrm{g}$ foram encontrados por Amaya et al. (2013b) quando utilizaram somente suco de manga em substituição à água.

Em estudo realizado por Branco et al. (2003), ao avaliar medidas físicas e químicas de suco de manga encontrou valor de 4,28 para $\mathrm{pH} ; 17,0 \%$ de sólidos solúveis e 0,89\% de fibras. A composição química dos sucos sugerem que sejam potenciais enfraquecedores da rede de glúten, reduzindo o volume específico dos pães.

A figura 4 apresenta a superfície de resposta e a curva de contorno da dos sucos de acerola e manga sobre a densidade dos pães tipo forma. 

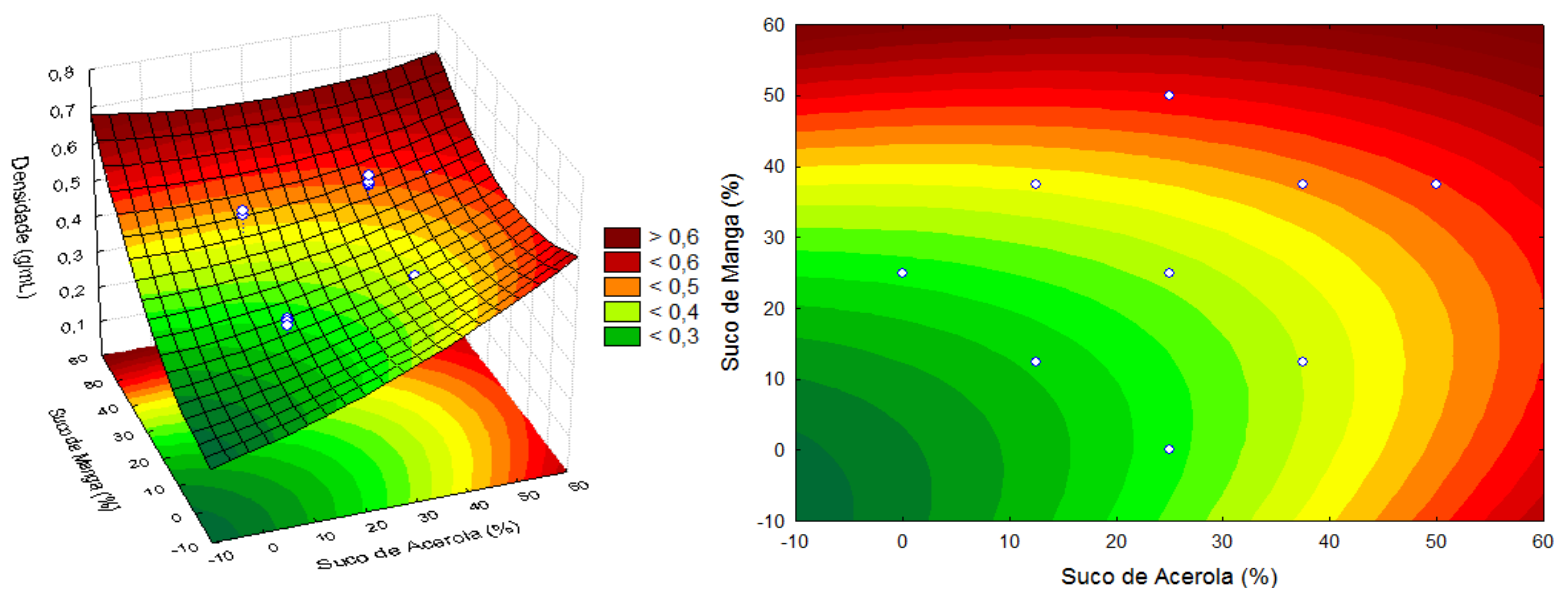

Figura 4 - Superfície de resposta, curva de contorno da ação dos suco de acerola e manga sobre a densidade dos pães

A densidade dos pães variou de $0,29 \mathrm{~g} / \mathrm{mL}$, para o ensaio 5 a $0,53 \mathrm{~g} / \mathrm{mL}$ (ensaio 4), de acordo com a superfície de resposta gerada, a zona de mínimo foi para valores de densidade inferiores a $0,4 \mathrm{~g} / \mathrm{mL}$, correspondente à adição de até $25 \%$ de suco de manga e $25 \%$ de suco de acerola.

Amaya et al., (2013b) estudaram a incorporação de 50\% e 100\% de suco de manga em substituição a água em formulações de pães, encontrou valores de densidade acima de 0,52 $\mathrm{g} / \mathrm{mL}$, obtendo $0,72 \mathrm{~g} / \mathrm{mL}$ quando a substituição foi total, a adição conjunta do suco de acerola com o de manga, melhorou a densidade dos pães quando comparados com este estudo, produzindo pães de melhor qualidade.

A figura 5 fornece a superfície de resposta e curva de contorno da ação dos sucos de acerola e manga sobre o índice de expansão dos pães.
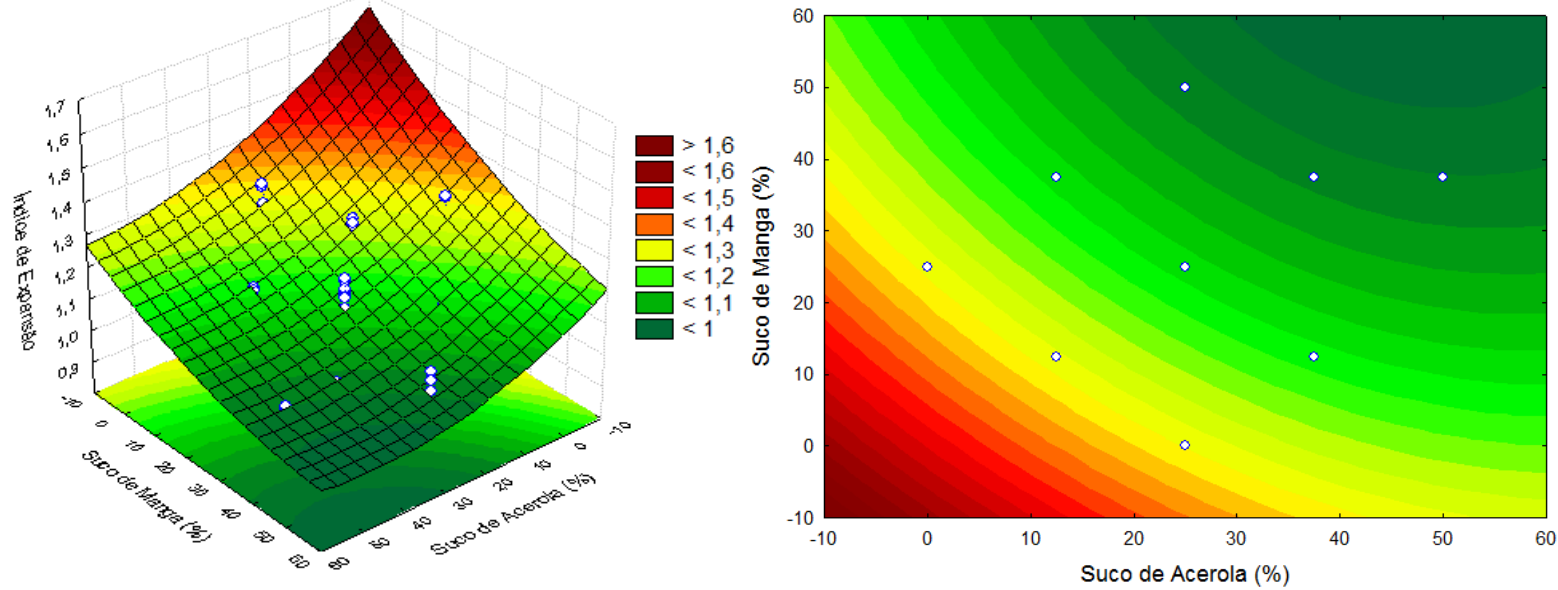

Figura 5 - Superfície de resposta e curva de contorno da ação do suco de acerola e manga sobre o índice de expansão dos pães

O índice de expansão apresentou os menores valores nos ensaios 4, 6 e 8, com 1,02; 1,02 e 1,05, respectivamente, não havendo diferenças significativas entre eles, ensaios estes, que foram adicionados de maior quantidade de suco de frutas, comprovando que há prejuízo à 
capacidade de expansão dos pães. O maior índice de expansão obtido foi de 1,32 no ensaio 5, quando foi adicionado apenas $25 \%$ de suco de manga, sem a incorporação de suco de acerola. Amaya et al., (2013c) avaliaram a substituição de água por suco de goiaba em formulações de pães tipo forma, onde verificaram que houve redução nos parâmetros de expansão das massas, entretanto, em maior intensidade do que o observado neste presente estudo.

\subsection{CONCLUSÃO}

Verificou-se que a adição de suco de acerola e manga em formulações tipo forma reduz os parâmetros físicos de qualidade dos pães tipo forma, sendo as condições ótimas de até $16 \%$ de suco de acerola e $18 \%$ de suco de manga não promovem prejuízos signifcativos aos produtos.

\subsection{REFERENCIAS}

AHMED, J.; RAMASWAMY, H. S.; HIREMATH, N. Effect of high pressure treatment on rheological characteristics and colour of mango pulp. Intern. J. Food. Sci. and Tech, v. 40, p. 885-895, 2005.

AMAYA, D. A. D.; PINHEIRO, G. K.; BRASIL, D. L.; MELO, P. E. F.; ZAMBELLI, R. A. Efeito do suco de caju na capacidade de expansão de massas durante o processo de fermentação. Magistra, v. 25, p. 482-487, 2013a.

AMAYA, D. A. D.; PINHEIRO, G. K.; BRASIL, D. L.; MELO, P. E. F.; ZAMBELLI, R. A. Incorporação de suco de manga em formulações de pães tipo forma: avaliação das características físicas. Magistra, v. 25, p. 436-440, 2013 b.

AMAYA, D. A. D.; GOES, T. S.; PINHEIRO, G. K.; BRASIL, D. L.; ZAMBELLI, R. A. Influência do suco de goiaba na capacidade de expansão das massas para a produção de pães tipo forma. Magistra, v. 25, p. 582-588, 2013c.

AMERICAN ASSOCIATION OF CEREAL CHEMISTS - A. A. C. C. Approved methods of American Association of Cereal Chemists. 9 ed. St. Paul: 1995.

BRANCO, I. G.; GASPARETTO, C. A. Aplicação da metodologia de superfície de resposta para o estudo do efeito da temperatura sobre o comportamento reológico de misturas ternárias de polpa de manga e sucos de laranja e cenoura. Ciência e Tecnologia de Alimentos, v. 23, n. 1, p. 166-171, 2003.

DE ROSSO, V. V.; HILLEBRAND, S.; MONTILLA, E. C.; BOBBIO, F. O.; WINTERHALTER, P.; MERCADANTE, A. Z. Determination of anthocyanins from acerola (Malpighia emarginata DC.) and açai (Euterpe oleracea Mart.) by HPLC-PDA-MS/MS. J. Food Composit. Analys, v. 21, p. 291-299, 2008.

GABRIC, D.; BEN-AISSA, F.; LE-BAIL, A.; MONTEAU, J. Y.; CURIC, D. Impact of process conditions on the structure of pre-fermented frozen dough. J. Food Eng. n.105, p. 361-366, 2011. 
MERCALI, G. D.; SCHWARTZ, S.; MARCZAK, L. D. F.; TESSARO, I. C.; SASTRY, S. Ascorbic acid degradation and color changes in acerola pulp during ohmic heating: effect of eletric field frequency. J. Food Eng, v. 123, p. 1-7, 2014.

MUSSER, R. D. S.; LEMOS, M. A.; LIMA, V. L. A. G.; MELO, E. D. A.; LEDERMAN, I. E.; DOS SANTOS, V. F. Características físico-químicas de acerola do banco ativo de germoplasma em Pernambuco. Ciência de Tecnologia de Alimentos, v. 24, p. 556-561, 2004.

RENUKA, B.; KULKARNI, S. G.; VIJAYANAND, P.; PRAPULLA, S. G.

Fructooligosaccharide fortification of selected fruit juice beverages: effect on the quality characteristics. Food Sci. and Technol, v. 42, p. 1031-1033, 2009.

SILVA, M. R.; GARCIA, G. K. S.; FERREIRA, H. F. Caracterização química, física e avaliação da aceitação de pão de queijo com baixo teor energético. Alim. e Nutr, v. 14, n. 1, p. 69-75, 2003.

SINGH, R. K.; SINGH, R. N. Effect of post-harvest treatments on shelf life of mango (Mangifera indica L.) fruits cv. Amrapali. Res. J. Agric. Sci, v. 1, p. 415-418, 2010.

WANG, J.; ROSELL, C. M.; BARBER, C. B. Effect of the addition of different fibres on wheat dough performance and bread quality. Food Chem., v. 79, n.2, p. 221-226, 2002. 